\title{
Alternative causal explanations for order break the link between order and agents
}

\author{
Adena Schachner, Minju Kim
}

Department of Psychology, University of California, San Diego

Corresponding author: Adena Schachner (email: schachner@ucsd.edu),

Department of Psychology, University of California, San Diego, 5111 McGill Hall, 9500

Gilman Drive, La Jolla, CA 92093-0109.

Minju Kim (email: minjukim@ucsd.edu), Department of Psychology, University

of California, San Diego, McGill Hall, 9500 Gilman Drive, La Jolla, CA 92093-0109.

Word Count: 9,511 


\begin{abstract}
People often believe that orderly structures were created by agents. We examine the cognitive basis of this tendency, and its malleability, focusing on orderly musical sounds as a case of interest. If simple associations mediate the link between order and agents, then detection of orderly stimuli should lead people to infer the presence of agents, independent of other factors. If causal reasoning mediates the link, then an alternative physical explanation for order should explain away its link with agents. We presented participants with orderly or disorderly outcomes in the form of musical sound sequences, and manipulated whether there was an alternative physical explanation for each outcome. Participants saw a scene involving a staircase-like xylophone, where a ball rolling downhill would produce an orderly descending scale. This provided an alternative physical explanation for how this orderly outcome could be generated. We found that when this scene was shown, then occluded, people did not infer the presence of an agent after hearing the orderly descending scale. In contrast, people expected an agent after hearing a scrambled sequence of tones, which could not be produced by gravity in this context (Exp. 1). When we subtly changed the scene by shuffling the xylophone's bars, so gravity would not create an orderly outcome, participants readily inferred the presence of an agent from the same orderly stimulus. When we controlled for the presence of an alternative explanation, and manipulated orderliness, orderliness had no effect (Exp. 2). Across both experiments, alternative causal explanations flipped participants' judgments such that disorder, and not order, led people to infer the presence of an agent. These findings show that the link between order and agents is mediated by causal reasoning, and can be weakened by understanding an alternative mechanism by which order could arise.
\end{abstract}

Keywords: social cognition; causal reasoning; order; pattern; agency; music 


\section{Introduction}

The visible order of the universe proclaims a supreme intelligence.

— Jean-Jacque Rousseau, Emile, 1762

Our human environments are full of orderly, complex structures: We hear orderly sounds, like music (Cross, 2003); create objects with orderly structure, like tools (Vaesen, 2012); and see order in nature (Paley, 1802; Dawkins, 1986). How do we reason about this 'orderliness', pattern or regularity?

People readily invoke intentional agents - both physical and supernatural - to explain the patterns and regularities they observe (e.g. Dawkins, 2006; Swinburne, 1968), and recent work has shown that this tendency arises early in life (Baer, Tam, Nancekivell \& Friedman, 2013; Friedman, 2001; Ma, Berthiaume, Hoch, \& Xu, 2013; Ma \& Xu, 2013; Newman, Keil, Kuhlmeier, \& Wynn, 2010). In effect, we appear to frequently and perhaps obligatorily link orderly outcomes with intentional, animate agents - whether these agents be human, animal, or supernatural. This link has broad consequences: For example, it helps explain the intuitive nature of creationist beliefs, as these involve positing a supernatural agent to explain order in nature (Barrett, 2007; Bering, 2011; Kelemen, 2004; Keil \& Newman, 2015).

What underlying cognitive processes cause us to link order with agents? This goes beyond the question of how we detect randomness versus regularity/pattern (Reimers, Donkin \& LePelley, 2018; Griffiths, Daniels, Austerweil \& Tenenbaum, 2018), to ask: Given that one has detected regularity, what leads us to believe it was produced by an 
ORDER, AGENTS AND ALTERNATIVE EXPLANATIONS

agent, versus an inanimate force (i.e. a non-random but inanimate process)? Two broad types of representations could underlie the link.

\section{Associations with perceptual features}

First, the link could be driven by a network of associations between animate agents and the perceptual features of orderly stimuli (Hamlin \& Baron, 2014; Keil \& Newman, 2015). Such associations could be learned from experience: We often observe other people organizing objects, and producing orderly things like music and art. We do not often observe inanimate objects or forces producing orderly outcomes. With experience, then, we should come to associate people with the orderly outcomes we have observed. We could then generalize from people to 'agents' as a class, as is common from early in life (Gelman \& Opfer, 2007); and generalize from the specific orderly outcomes we have seen to other perceptually similar outcomes. A nativist version of this account is also possible: There could be an innate, hard-wired association between the detection of orderly outcomes and the concept 'agent' (Keil \& Newman, 2015; Bering 2011). Such a mechanism would allow us to detect other agents' recent presence, an ability with clear adaptive value (Atran \& Norenzayan, 2005; Boyer, 1994; Vallortigara, 2012).

Whether the associations are innate or learned, these accounts posit that agents are associated with the percept of orderly stimuli, such that the detection of orderliness will cue expectations of agency. This idea has been used to explain a range of phenomena across multiple literatures. In music cognition, an association between orderly sounds and people has been used to argue that musical patterns could evoke agency and social concepts in an automatic or obligatory way (Launay, 2015). In religious cognition, 
associations between order and agents have been used to explain why beliefs in a supernatural creator are highly intuitive and persistent, while physical-mechanical explanations of order in nature (i.e., evolution by natural selection) are counterintuitive (Barrett, 2007; Bering, 2011; Cornelius \& Lopez-Mobilia, 2013; Kelemen, 2004; Kelemen, 2012). Overall, associations provide an account of the order-agent link that is both plausible and influential.

\section{Causal reasoning (rational inference)}

In contrast to this associative account, the link between agency and order may arise as a product of causal reasoning about how the orderliness was generated, through a process of inference to the best explanation (Lipton, 2004; Keil \& Newman, 2015; Ma \& Xu, 2013; Tenenbaum, Griffiths \& Kemp, 2006). Under this account, to explain observed data (i.e. an orderly outcome), people consider multiple hypotheses about its cause (perhaps it was generated by a person; by an animal; by gravity; by wind; etc.). They then choose the hypothesis that is both plausible a priori and provides a good explanation of the observed data. This type of inferential reasoning occurs in multiple related domains, including physical-mechanical reasoning, social reasoning, and mental state inference (Teglas et al., 2011; Baker et al., 2009; Battaglia, Hamrick \& Tenenbaum, 2013).

If causal reasoning drives the link between order and agents, then such processing should not be obligatory or automatic. Instead, the presence of an alternative causal explanation should make an orderly stimulus weaker evidence of agents - even for the very same perceptual stimulus. That is, knowledge of a plausible physical-mechanical cause should 'explain away' orderliness, reducing the likelihood that people infer an agent as cause. This contrasts with the prediction of the simpler associative account 
above: Only a structured system of causal inference would allow one alternative explanation to weaken evidence of other explanations. Such 'explaining away' is a signature of inferential causal reasoning, and serves as evidence that the underlying cognitive representation is rich and structured (Gopnik \& Sobel, 2000; Pearl, 2000; Tenenbaum et al., 2006).

\section{Teasing apart the two accounts}

The current studies aim to tease apart these two accounts in adult cognition, asking whether the link between order and agents is driven by associations, causal reasoning, or both processes simultaneously. In doing so, we probe the malleability of the link between agency and order (Meng, Griffiths \& Xu, 2017): Can we weaken or break the link between order and agents, by understanding alternative causal mechanisms by which order could arise? If causal reasoning accounts for the link, we would expect it to be malleable in this way; if a simpler association accounts for the link, we would expect it to be robust in the face of conflicting high-level causal knowledge.

To tease apart the two theories, we presented participants with orderly or disorderly outcomes, and manipulated whether there was an alternative physicalmechanical explanation for each outcome (i.e. whether it could be produced by gravity alone). We asked if this alternative explanation could 'explain away' orderliness, eliminating participants' tendency to infer the presence of agents from observations of order. To provide a clear test, we pit the two accounts against one another, setting up a situation where causal reasoning predicts that disorder will be linked with agents while order will not - leading us to predict a complete flip in the usual reported results. 
ORDER, AGENTS AND ALTERNATIVE EXPLANATIONS

\section{Orderliness in music, and reasoning about the causes of sounds}

The current experiments focus on musical sounds as a case study of orderly stimuli: Previous work has shown that orderly sounds cue agency, just as orderly visual stimuli do (Ma et al., 2013; Launay, 2015). Musical sounds provide a particularly ecologically valid and relevant case: Music is universally present across cultures (Brown, 1991), and music itself is often defined in terms of orderliness, as "organized sound" (Blacking, 1973).

Music also presents a domain where evidence of high-level causal reasoning would have important theoretical implications. In music cognition, one major question of interest is why music and movement are linked (Changizi, 2011; Grahn \& Brett, 2007; Hannon, Schachner \& Nave-Blodgett, 2017; Repp \& Su, 2013; Sievers, Polansky, Casey, \& Wheatley 2013; Thaut et al., 1996). Previous work has theorized that musical sounds are linked with movement due to low-level, modality-specific connections between auditory and motor representations (Chen, Penhune \& Zatorre, 2008; Grahn \& Brett, 2007; Patel, Iversen, Chen, \& Repp, 2005). The current experiments serve to test a novel hypothesis: Musical sounds may activate representations of agents and actions through high-level causal reasoning, which may support abstract inferences about the actions, actors, and objects involved in causing sounds. In particular, since all sounds occur through movement, people could infer what kinds of movements occurred to cause the sounds; these movements would inform whether an animate agent caused the sounds. For example, if producing the sounds would require changing direction in a self-propelled way, then an agent would be the most likely cause. 
ORDER, AGENTS AND ALTERNATIVE EXPLANATIONS

Such a finding would provide evidence that high-level causal inference, not just low-level perceptual-motor representations, links music to movement and to abstract social concepts. This would inform understanding of how music activates social cognitive brain mechanisms and representations of action (Changizi, 2011; Grahn \& Brett, 2007; Overy \& Molnar-Szakaks, 2009; Sievers, Polansky, Casey, \& Wheatley, 2012), and also inform understanding of the cognitive processes underlying auditory event perception (Bregman, 1990; Gaver, 1993; Seigel, Tenenbaum, \& McDermott, 2018; Gerstenberg, Siegel \& Tenenbaum, 2018).

\section{The current studies}

The current studies thus probed the cognitive basis by which orderly stimuli are linked with agents, focusing on musical sounds as a particular domain of interest. In our experiments, participants saw a scene involving a staircase-like xylophone (Figures 1,3). In Experiment 1, the xylophone's bars were positioned normally, such that a ball simply rolling down the xylophone would produce a descending scale - a highly ordered sequence of sounds (as verified in an independent sample; see below). In this context, inanimate forces and objects (gravity, the ball) provide an alternative explanation for how the descending scale could plausibly be generated. If causal reasoning mediates the link between order and agents, then the descending scale should not lead people to expect an agent in this context, in spite of its orderliness. In contrast, a scrambled sequence of tones

should lead people to expect an agent - because producing these notes would require selfpropelled movement in this context. Thus in Experiment 1, we predict results that are flipped from what is typically reported: Disorder, and not order, should lead people to 
infer the presence of an agent in this context. In contrast, if a network of associations between percepts of order and agents drive the link, then orderly sounds should lead observers to expect an agent, even in this context.

A second experiment (which was preregistered) provided an additional novel test of the associative and causal reasoning accounts. We probed an additional outcome - an orderly ascending scale - comparing it to the descending scale and scrambled tones. In the context of the normal, bars-descending xylophone, both the ascending scale and the scrambled tones lack an alternative physical explanation: Gravity would not allow the ball to produce either one. Thus the causal reasoning account predicts that agency judgments should be equivalent for these two outcomes, when they occur in the context of a normal xylophone. However, if associations drive people to expect agents when they perceive order, then participants should expect an agent more strongly for the ascending scale than the scrambled tones condition. In this way, this comparison between the ascending scale and scrambled tones allowed us to equate for the lack of an alternative physical explanation, while manipulating orderliness, to examine these effects separately.

In the second experiment we also compared the normal xylophone to a new context, in which the xylophone's bars appeared in scrambled positions, as if they had been switched around (Figure 3). On this new bars-scrambled xylophone, rolling downhill would generate a scrambled sequence of tones, not an orderly descending scale. This condition provides an analogue to the situations explored in previous work, in which inanimate forces cannot produce order (e.g. Newman et al., 2010). If causal reasoning can drive the link between order and agents, and also explain it away, then the orderly descending scale should lead people to expect an agent in the context of this new 'bars- 
ORDER, AGENTS AND ALTERNATIVE EXPLANATIONS

scrambled' xylophone — and not in the normal xylophone context. In contrast, if a network of associations between percepts of order and agents drive the link, then orderly sounds should lead observers to expect an agent across both contexts.

\section{Experiment 1}

In a first experiment, participants viewed a staircase-like xylophone, where the xylophone's bars were positioned such that a ball rolling down the staircase would produce a descending scale, a highly orderly sequence of sounds. We asked whether participants expected only agents to produce the orderly descending scale, as well as a scrambled sequence of the same tones - which was less orderly, but could not be generated by physical causes like gravity in this context.

To probe participants' expectations about agents, we used two complementary measures. First, we measured participants' levels of surprise after a ball or agent appeared to produce order or disorder. Second, we measured whether participants changed their beliefs about the ball's (or agents') agency after observing them appear to produce order or disorder, e.g. by concluding that the ball was actually alive.

If people's judgments rely on a network of associations between the percept of order and agents, then participants should be surprised when an inanimate ball appears to produce the orderly outcome, as this violates the association. Afterward, they should also judge the ball more likely to be alive/animate than they had previously, since it appeared in association with order.

In contrast, if causal reasoning mediates the link between order and agents, then the orderly descending scale should not lead observers to expect an animate agent in this 
context. As such, we predicted that participants would not be surprised when an inanimate ball seemed to produce the orderly outcome, and would not judge the ball more likely to be animate/alive after this observation. Instead, because the disorderly scrambled tones have no alternative physical explanation in this context, the disorderly scrambled tones should lead observers to expect an agent. We thus predicted that participants would be surprised, and afterward judge the ball more likely to be animate/alive, after it seemed to produce disorder - and not after it seemed to produce the orderly descending scale.

\section{Method}

Each participant took part in one of four between-subject conditions, with one of two apparent causes (inanimate ball or animate agent), and one of two sound sequences as the outcome (orderly descending scale or less-orderly scrambled tones). All stimuli and the raw dataset are available at https://madlab.ucsd.edu/orderAndAgents.html, and as supplemental materials.

To verify that the sounds of the descending scale were perceived as more orderly than the scrambled tones, we conducted a separate study as a manipulation check, asking participants to judge which of the two sound outcomes was "more orderly, or organized". The descending scale was chosen as "more orderly" by 19 out of 20 unique participants, none of whom participated in the main experiment ( $p<0.0001$, binomial test).

Participants. 160 adult residents of the United States (18+ years of age) took part in the experiment over the internet, via the Amazon Mechanical Turk website (MTurk, https://www.mturk.com). Sample size was determined through a separate pilot study with 80 participants, which enabled us to estimate expected effect sizes. Based on this pilot 
ORDER, AGENTS AND ALTERNATIVE EXPLANATIONS

data, we determined that a sample of 160 participants ( $n=40$ per condition) would ensure adequate power on our primary measures (0.7-0.9). Participants were required to have had at least $93 \%$ of their previous work on the MTurk website judged as acceptable; this criterion was determined a-priori based on criteria used in the literature (Berinsky, Huber \& Lenz, 2012; Goodman, Cryder \& Cheema, 2012; Mason \& Suri, 2011). All participants gave informed consent, and were paid $\$ 0.50$ for approximately 5 minutes of their time. Repeat participation by the same individual was not allowed (each participant was identified by a unique and stable code by the MTurk system). 9 additional participants were run, but excluded from the final sample for getting the pitch perception/ memory check questions wrong (7 participants), leaving questions blank (1), or having technical difficulties that prevented viewing the videos (1). 20 additional unique participants took part in a separate study on MTurk to determine the relative orderliness of the stimuli (see above), gave informed consent and were paid $\$ 0.15$ each for $\sim 1 \mathrm{~min}$ of their time.

Stimuli. Animated videos and accompanying sounds were constructed using Apple Keynote '09, Apple GarageBand '09, and Apple QuickTime Pro 7 software. A first video introduced either the animate agent or the inanimate ball, depending on condition (matched for length; 15s each). These videos showed that the agent was animate, and that the ball was inanimate, by including known cues to animacy or inanimacy (Gelman \& Opfer, 2007; Johnson, 2003; Tremoulet \& Feldman, 2000). In the agent video, the agent moved on its own, had a face, and seemed to respond to its environment; in the ball video, the ball rolled as if by gravity (Figure 1). 
ORDER, AGENTS AND ALTERNATIVE EXPLANATIONS

A second video introduced the staircase-like xylophone, and demonstrated how it produced sound (11s). The video showed the staircase with colored bars; a mallet then entered and played a sequence of sounds before exiting the scene. This sound sequence was designed so that the sounds played at test would each be equally novel relative to the sounds participants had previously heard in the experiment, and consisted of an ascending scale (1-2-3-4-5) and a five-note melody (5-2-4-3-1). The sounds indirectly showed that the stairs were arranged to play the following pitches from left to right: $G, F$, E, D, C (or 5, 4, 3, 2, 1, with fundamental frequencies of 778, 702, 647, 569, and 511 $\mathrm{Hz})$.

During the test trial, an additional video was shown (10s) in which the xylophone was covered by a black square; the ball or agent entered the scene from the top of the hill and rolled behind the occluder, and one of two sound outcomes played: a descending scale (5-4-3-2-1) or a scrambled sequence of the same tones (2-5-3-4-1). These tone sequences were of equal duration, and were equally novel relative to the sounds participants had previously heard in the experiment (both had one two-note sequence in common with the video introducing the xylophone, 4-3 or 3-4).

Procedure. To ensure that all participants' computers could play sounds, participants were first asked to identify two simple sounds. Participants clicked a link to submit these answers, and move to a second page. Throughout the experiment, participants could not return to previous pages after moving forward to a new page, preventing revision of answers based on subsequent information.

On the second page, participants began the main task. They were instructed to view a video of the ball or agent (depending on condition), and were asked to describe 
what happened. They then judged whether the ball or agent was animate/alive, on a -2 to +2 scale ("Do you think this is meant to represent a living thing, or an inanimate object?"; Definitely a living thing, Probably a living thing, Could be either one, Probably an inanimate object, Definitely an inanimate object). Participants were asked to explain their answer ("Why?", free-response answer), before moving to a third page.

On the third page, participants viewed the xylophone demonstration video, and were asked to describe what happened. To ensure participants distinguished the multiple pitches and remembered how they mapped to the bars of the xylophone, we asked two multiple-choice memory check questions ("Which part of the staircase plays the highest note?"; "Which part of the staircase plays the lowest note?"). Participants then moved to a fourth page.

Participants then completed one test trial: They viewed a video where the xylophone was covered; the ball or agent entered and rolled behind the occluder, and one of two sound outcomes played (descending scale or scrambled tones). Participants were then asked to judge how surprising the sounds were, on a 1-4 scale ("Were the sounds surprising? The sounds were: not surprising, a little surprising, somewhat surprising, very surprising"). They were asked to explain their answer ("Why/why not?"), and to describe what happened in the video, before moving to a final page. On the last page, participants were asked to judge whether the ball or agent was alive/animate, on the same 1-5 scale as before (see above). They were asked to explain their answer ("Why?"), and to guess what the experiment was about, before submitting their answers. 
Task:
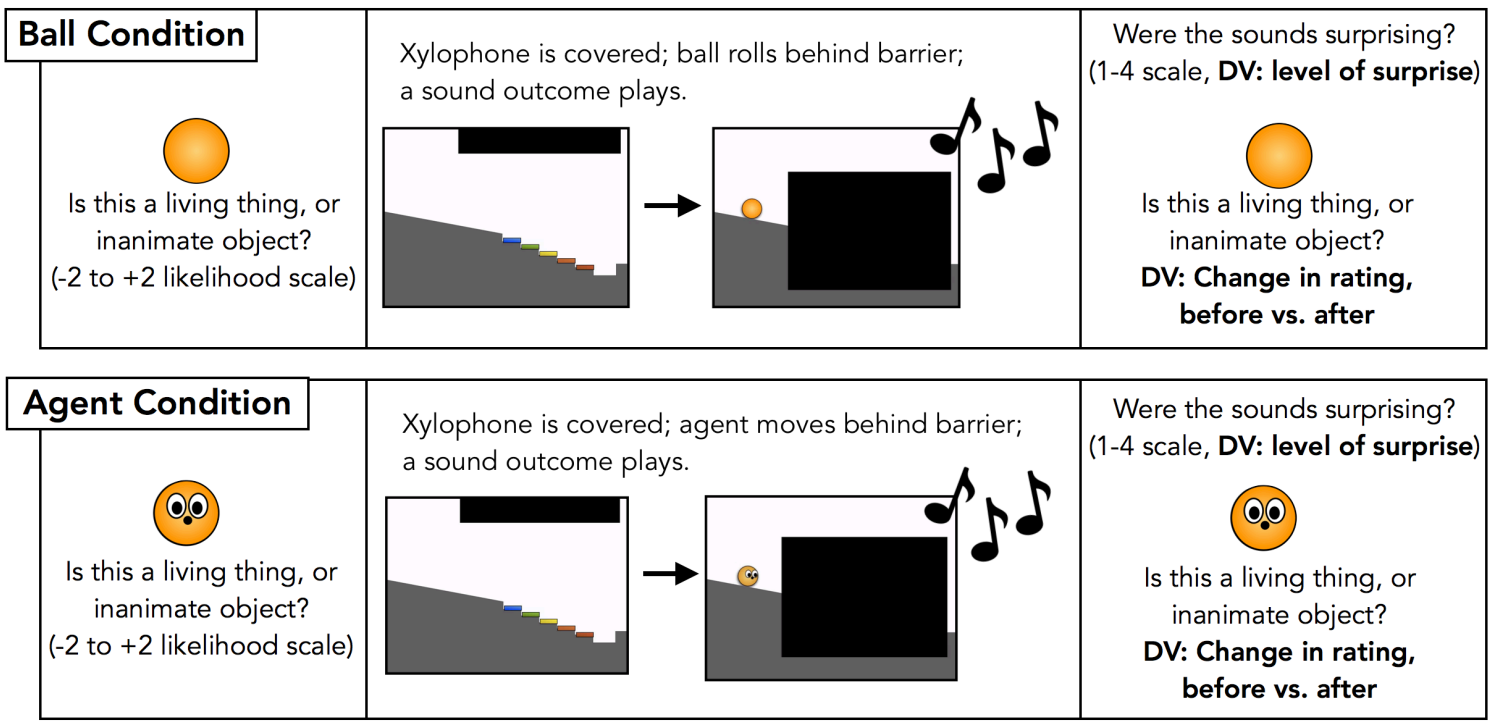

Two sound outcomes (orderliness manipulation)
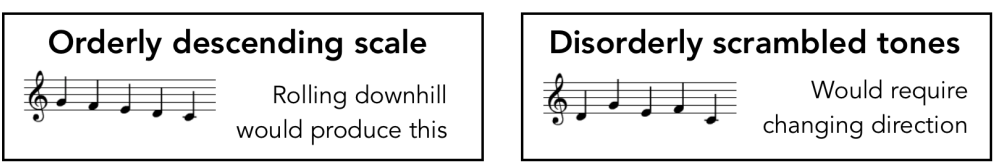

Figure 1. Method. We showed participants either an inanimate ball or an animate, agentic character, which appeared in the context of a staircase-like xylophone. The xylophone was covered, the ball or agent rolled behind the barrier, and one of two sound outcomes occurred: An orderly descending scale, or disorderly scrambled tones. Crucially, the xylophone's position was such that a ball rolling downhill would produce an orderly descending scale; thus it provided a physical-mechanical explanation for the orderly descending scale. To measure expectations about agents, we measured participants' levels of surprise after each outcome, and whether they changed their beliefs about agency, e.g. by concluding that the ball was actually an agent. The associative account predicts that detection of orderliness should cue agency even in this context, while causal reasoning predicts that the disorderly outcome, and not the orderly outcome, will be linked with agents in this context.

\section{Results}

Our intended animacy manipulation was successful: The vast majority of participants initially rated the ball as inanimate (76/ 80 rating as definitely or probably inanimate, $p<0.0001$, binomial test; $M=-1.56$ on a -2 to +2 scale, $S E M=0.07, p<0.0001$, two-tailed t-test), or rated the agent as alive (62/ 80 rating as definitely or probably alive; $p<0.0001 ; M=0.93, S E M=0.13, p<0.0001)$. 
We compared participants' animacy judgments before versus after observing each sound outcome, asking which outcome led participants to change their judgments about the ball's animacy. In contrast with the predictions of the associative account, participants did not judge the ball more likely animate after it seemed to produce the orderly descending scale (Mean change $=0.05, S E M=0.15, d=0.05 ; p=0.74)$. Instead, in line with the causal reasoning account, participants judged the ball more likely to be animate after it seemed to produce the disorderly scrambled tones, which would require self-propelled movement to produce in this context (Mean change $=0.68$ on a -2 to +2 scale, $S E M=0.18, d=0.58 ; p<0.001$, two-tailed t-test against zero change; these levels of change were different from one another; $d=0.59, p=0.01$; see Figure 2).

We next examined participants' judgments of whether each outcome had been surprising. We predicted that if participants' expectations were driven by causal reasoning, there should be an interaction between apparent cause and sound outcome, such that participants would be more surprised when the ball seemed to produce the scrambled tones than the orderly descending scale; and that this difference in surprise would be lessened when the agent was present. To examine this prediction, we conducted a 2 (apparent cause: ball vs. agent) by 2 (sound outcome: descending scale vs. scrambled tones) ANOVA with participants' surprise rating as the dependent measure. As predicted, we found a significant interaction between apparent cause and sound outcome, $F(1,156)=7.53, p<0.01, \eta_{p}^{2}=0.05$, as well as main effects of apparent cause $(F(1$, $\left.156)=5.66, p=0.019, \eta_{p}^{2}=0.04\right)$ and sound outcome $(F(1,156)=101.27, p<0.0001$, $\left.\eta_{p}^{2}=0.39\right)$. In line with the causal reasoning account, participants were more surprised when the ball produced the disorderly scrambled tones $(M=2.93$ on $1-4$ scale, $S E M=0.17)$ 
than when it produced the orderly descending scale $(M=1.18, S E M=0.09 ; p<0.0001$, twotailed t-test). This was not just because the scrambled tones were always surprising: Participants were more surprised when the ball produced the scrambled tones than when the agent produced them $(M=2.23, S E M=0.17$; Mean difference $=0.7$ on $1-4$ scale, $d=0.65$; $p<0.01$, two-tailed t-test). Participants also did not show any expectation that only agents would produce order: They were equally unsurprised by the orderly descending scale whether it was produced by the agent $(M=1.18, S E M=0.09)$ or ball $(M=1.23, S E M=0.09$; Mean difference=-0.05; $p=0.70, d=0.09$; two-tailed t-test; see Figure 2). ${ }^{1}$

a. Change in animacy rating of ball or agent, before vs. after hearing each sound

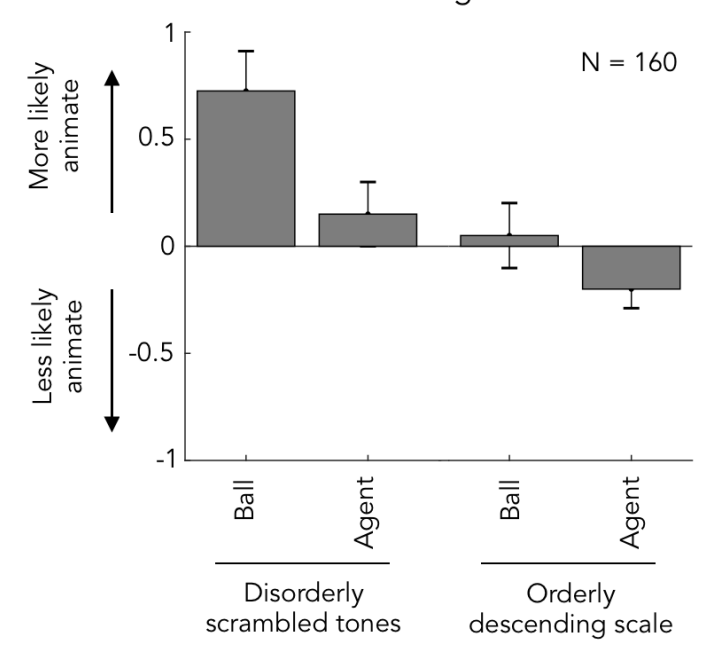

b. Surprisingness of the sounds,

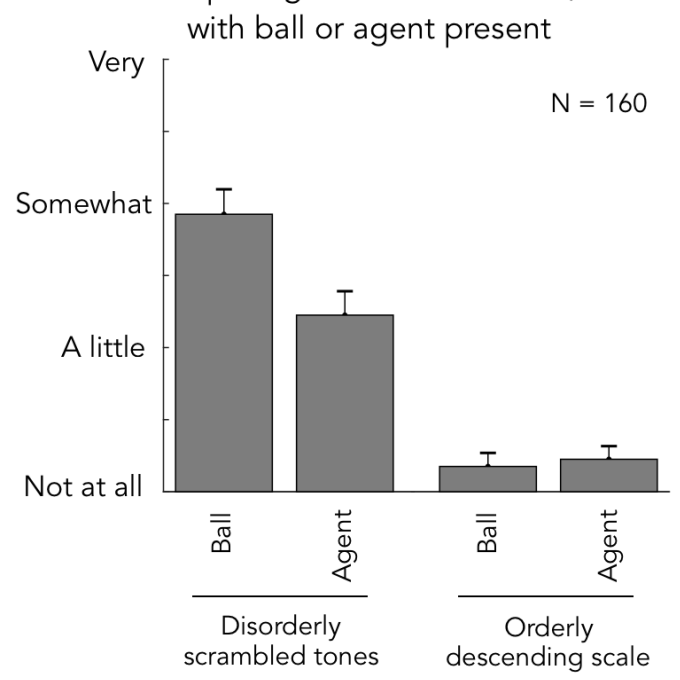

Figure 2. Results. Participant's judgments of animacy and surprise, after observing a ball or agent seem to produce an orderly descending scale or disorderly scrambled tones, in a context that provided a physical-mechanical explanation for the orderly outcome (and not for the disorderly outcome). (A) Participants did not judge the ball more likely to be animate after it seemed to produce the orderly descending scale; they instead judged the

\footnotetext{
${ }^{1}$ Findings remain the same when data are analyzed using nonparametric tests (ordinal logistic
} regressions, rank-sum tests). 
ball more likely animate after it appeared to produce disorderly scrambled tones. (B) Participants were not surprised when the ball produced the orderly descending scale, and were most surprised when the ball produced the disorderly scrambled tones. Disorder, not order, was linked with agency in this context, suggesting that the link between order and agents is moderated by causal reasoning. Error bars are standard error of the mean.

\section{Discussion}

These data provide initial evidence that the link between order and agents is malleable, and is modulated by causal reasoning. Participants saw a scene involving a staircase-like xylophone, where a ball simply rolling down the xylophone would produce an orderly sound sequence - a descending scale. This provided an alternative physicalmechanical explanation for how the orderly descending scale could be generated. We found that the descending scale did not lead people to expect an agent in this context, in spite of its orderliness. In contrast, a scrambled sequence of the same tones - which would require self-propelled movement to produce in this context - did lead people to expect an agent.

We thus find that when participants have an alternative explanation for how order could have been generated, this can flip their judgments from what has been typically reported: Disorder, and not order, led people to infer the presence of an agent. This suggests that causal reasoning moderates the link between order and agents, and that associations between percepts of order and agents may not play a major role: If a network of associations between percepts of order and agents drove the link, then orderly sounds should have led observers to expect an agent, even in this context.

Two important questions remain to be addressed. Firstly: What if both types of processes (causal reasoning, and associations between the percept of order and agents) are occurring and affecting judgments simultaneously? Because our first experiment 
pitted these two accounts against one another, a larger effect of one process (causal reasoning) could have hidden or washed out a smaller effect of the other (associations).

A second experiment (which was preregistered) therefore provided a novel test, to detect simultaneous effects of associations and causal reasoning. We probed an additional outcome - an orderly ascending scale - comparing it to the descending scale and scrambled tones. In the context of the normal, bars-descending xylophone, both the ascending scale and the scrambled tones lack an alternative physical explanation: Gravity would not allow the ball to produce either one. Thus the causal reasoning account predicts that agency judgments should be equivalent for these two outcomes (in the normal xylophone context). However, if associations drive people to expect agents when they perceive order, then participants should expect an agent more strongly for the ascending scale than the scrambled tones condition. In this way, this comparison between the ascending scale and scrambled tones allowed us to equate for the lack of an alternative physical explanation, while manipulating orderliness, to examine these effects separately.

Secondly, in additional within-subject trials we also compared the normal xylophone to a new context, in which the xylophone's bars appeared in scrambled positions, as if they had been switched around (Figure 3). This condition provided an analogue to the situations explored in previous work, in which inanimate forces cannot produce order (e.g. Newman et al., 2010): Here, rolling downhill would generate a scrambled sequence of tones, not an orderly descending scale. If causal reasoning can drive the link between order and agents, and also explain it away, then the orderly descending scale should lead people to expect an agent in the context of this new 'bars- 
ORDER, AGENTS AND ALTERNATIVE EXPLANATIONS

scrambled' xylophone — and not in the normal xylophone context. This result would provide strong evidence that the 'flipped' results of Exp. 1 were due to the presence of an alternative explanation for the orderly outcome - rather than some other unknown aspect of our stimuli.

\section{Experiment 2}

The second experiment used a preregistered design to provide two tests of the role of causal reasoning and learned associations in linking agents and order. First, we compared an additional outcome - an orderly ascending scale - comparing it to the descending scale and scrambled tones. In the context of the normal, bars-descending xylophone, both the ascending scale and the scrambled tones lack an alternative physical explanation: Gravity would not allow the ball to produce either one. This allowed us to probe for simultaneous effects of associations and causal reasoning: If causal reasoning modulates the link, and associations also bias people to expect agents when they perceive order, then participants should expect an agent more strongly for the ascending scale than the scrambled tones condition.

Second, we compared two highly similar contexts: a normal xylophone (as in Exp. 1), and a new 'bars-scrambled' xylophone. In this new context, rolling downhill would generate a scrambled sequence of tones, not an orderly descending scale. If causal reasoning drives people to link order with agents, then the orderly descending scale should lead people to expect an agent in the context of this new 'bars-scrambled' xylophone - and not in the normal xylophone context. This would provide evidence that the 'flipped' results of Exp. 1 - in which order was not linked with agents - were due to 
ORDER, AGENTS AND ALTERNATIVE EXPLANATIONS

the presence of an alternative explanation for the orderly outcome, rather than some other unknown aspect of our stimuli.

Each participant completed six trials, with two contexts (normal and barsscrambled xylophones) combined with each of three sound outcomes (an orderly descending scale, disorderly scrambled tones, or an orderly ascending scale). On each trial, the scene was covered with an animated curtain, and a sound outcome occurred. Based on the sounds, we asked participants to judge whether an inanimate ball or animate agent had been present behind the curtain, rating the probability on a five-point scale.

\section{Method}

Study design, sample size, and analyses were pre-registered before data collection (accessible at https://aspredicted.org/blind.php? $\mathrm{x}=\mathrm{a}$ 3ii53). All stimuli and the raw dataset are available at https://madlab.ucsd.edu/orderAndAgents.html, and also as supplemental materials. The experiment followed a 2 (context) x 3 (sound outcome) within-subject design (see Figure 3). Each participant completed two blocks (by context: normal xylophone, bars-scrambled xylophone) of three trials each (sound outcome: descending scale, scrambled tones, ascending scale). The order of the blocks, and the order of trials within a block, were counterbalanced between subjects.

To verify that the new ascending scale stimulus was perceived as more orderly than the scrambled tones and equally orderly to the descending scale, we asked a separate sample of participants to listen to pairs of sound sequences, and judge which sound was more orderly or if the sounds were equally orderly. Participants judged the ascending scale as more orderly than the scrambled tones (16/20 participants; $p<0.01$, binomial test), and rated the descending scale and ascending scale as equally orderly ( $9 / 20$ saying 
ORDER, AGENTS AND ALTERNATIVE EXPLANATIONS

'equal', with others choosing each sound equally often, Ascending: 6/11; Descending: $5 / 11 ; \mathrm{p}=1.0)$

Participants. 60 adult participants were recruited from Amazon Mechanical Turk website and tested online, with the same requirement criteria and payment as Experiment 1 (37 male; Mean age=35.07, Range $19-61$ years). Sample size was pre-registered a priori. 34 additional participants were tested, but excluded following our preregistered exclusion criteria, for leaving questions blank (3 participants), failing to identify sounds in the initial sound check (1), or failing the pitch perception/ memory check questions (24; recalling exactly how each pitch mapped to each bar was likely more challenging for the bars-scrambled xylophone), or having participated in a previous similar experiment (6).

Stimuli. Stimuli were constructed using the same software as Experiment 1. Two videos demonstrated how each xylophone produced sound, one for each of the two contexts (normal and bars-scrambled xylophones). Both videos showed a similar scene: A staircase with colored bars, a pipe at the top of the hill, and a small 'digital display' on the top left corner of the video, used to scaffold pitch memory (this displayed a visual record of the notes that had been played, as numbers; see Figure 3). In both videos, a mallet entered the scene and played a sequence of sounds before exiting the scene.

The mallet moved in the same path and struck the same positions in both videos; this resulted in a different sequence of pitches across the two videos. The sequences were designed so that the sounds played at test would each be equally novel relative to the sounds participants had previously heard in the experiment. These videos thus included each of the test sequences one time, along with an additional five-note sequence. 
For the normal xylophone, the sounds were an ascending scale (1-2-3-4-5), two disorderly five-note sequences (5-2-4-3-1; then 1-3-4-2-5), and a descending scale (5-43-2-1). For the bars-scrambled xylophone, the sounds were a disorderly five-note sequence (5-2-4-3-1), an ascending scale (1-2-3-4-5), a descending scale (5-4-3-2-1), and another disorderly five-note sequence (1-3-4-2-5).

The sounds indirectly showed that for the normal xylophone, the stairs were arranged to play the following pitches from left to right: G, F, E, D, C (or 5, 4, 3, 2, 1, with fundamental frequencies of 778, 702, 647, 569, and $511 \mathrm{~Hz}$ ). For the bars-scrambled xylophone, the video indirectly showed that the bars were arranged to play C, E, F, D, G $(1,3,4,2,5)$.

During each test trial, one of six additional videos were played. In these videos, the xylophone and pipe were covered by an animated curtain, and one of three sound outcomes played: a descending scale (pitch sequence 5-4-3-2-1), an ascending scale (1-23-4-5), and a scrambled sequence of the same tones (1-3-4-2-5). All tone sequences were of equal duration.

Procedure. Participants were first asked to report age and gender, before clicking a link to submit these answers and move to a second page. Throughout the experiment, participants could not return to previous pages after moving forward to a new page, preventing revision of answers based on subsequent information. On a second page, participants were asked to identify two simple sounds, to ensure that all participants' computers could play sounds.

On the third page, participants began the main task: They were instructed to watch one xylophone demonstration video, and were asked to describe what happened. To 
check that participants remembered how the notes mapped to the bars of the xylophone, participants were asked two multiple-choice pitch perception/ memory check questions ("Which part of the staircase plays the highest note?"; "Which part of the staircase plays the lowest note?'). Participants then moved to a fourth page.

On the fourth page, participants were pointed to the pipe on the left-hand side of the scene, and were told that one of two things could come out of the pipe: An inanimate ball, or a cartoon character. To show the ball's inanimacy and the character's animacy, they were identified in ways consistent with inanimacy (as "a billiard ball") or animacy (as "a cartoon character"), and brief videos were shown of the character moving in a selfpropelled way, or the ball rolling with gravity (same videos as in Exp. 1). To emphasize animacy, the character was also labeled with a proper name (Fred).

Participants then completed a block of three test trials, each presented on a separate page. On each trial, participants watched a video where the scene was occluded, and a brief sound sequence played (the descending scale, scrambled tones, or ascending scale). Participants were then asked to judge: "What came out of the pipe? Was it the ball? Or Was it Fred [the cartoon character]?", on a -2 to +2 scale (Definitely the ball, Probably the ball, Could be either one, Probably Fred, Definitely Fred), and asked to explain their answer ("What made you think that?").

Participants then read similar instructions for the second block (the other context). They viewed the other of the two xylophone demonstration videos, and then completed the same questions and parallel three test trials as in the first block, but with videos showing the new context. Participants lastly were asked if the ideas in the questions were clear, and to guess what the experiment was about, before submitting their answers. 


\section{Task instructions:}

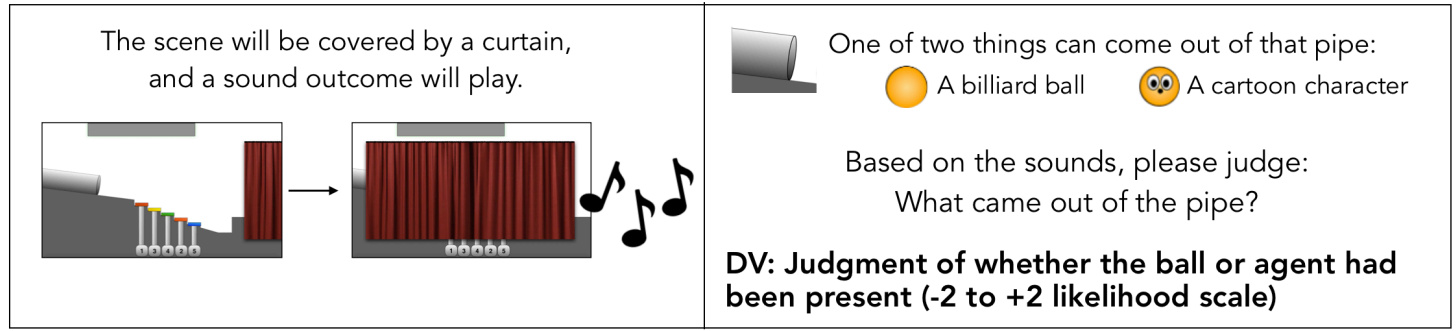

Three sound outcomes (orderliness manipulation)

Orderly Descending Scale

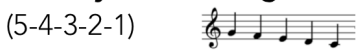

Disorderly Scrambled Tones $(1-3-4-2-5)$

Orderly Ascending Scale

(1-2-3-4-5) ל⿺辶. x Two xylophone contexts

(provides alternative physical-mechanical explanation)

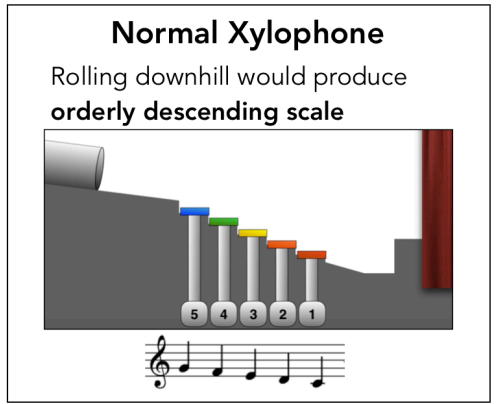

Bars-Scrambled Xylophone

Rolling downhill would produce scrambled tones

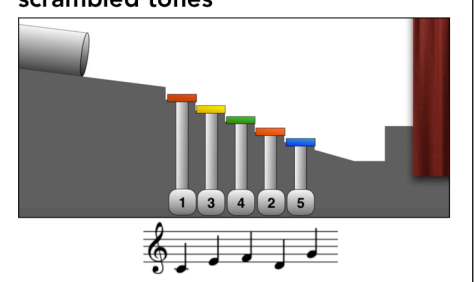

Figure 3. Method, Exp. 2. Participants were shown a scene with xylophone-like device; on each trial, the scene was covered with a curtain, a sound outcome occurred, and participants judged whether an inanimate ball or animate agent had been present behind the curtain. We tested three sound outcomes, which differed in their perceived orderliness. We also tested two different xylophone contexts, which allowed us to manipulate whether each sound could have been produced by the ball rolling downhill thus whether there was an alternative physical-mechanical explanation for the sounds.

\section{Results}

Following our pre-registered analysis plan, we examined participants' judgments of whether an agent or ball had been present, asking whether the orderliness of each sound outcome or the presence of an alternative explanation accounted for participants' judgments. We predicted that sounds would only lead participants to infer an agent when there was no alternative physical-mechanical explanation for how it occurred (see 
ORDER, AGENTS AND ALTERNATIVE EXPLANATIONS

Predictions, Figure 4). Thus, in the normal xylophone context, we predicted that the scrambled tones and the orderly ascending scale would lead people to infer agents, while the orderly descending tones would not. In the bars-scrambled xylophone context, we predicted that the descending tones and ascending tones would lead people to infer agents, while the scrambled tones would not.

To examine these predictions, we first probed for the predicted interaction between context and sound outcome with an omnibus test. We conducted a 2 (context: bars-descending vs. bars-scrambled xylophone) by 3 (sound outcome: descending scale vs. scrambled tones vs. ascending scale) repeated measures ANOVA with the rating of likelihood that the agent vs. ball was present as the dependent measure $(-2$ to +2 probability scale). As predicted, we found a significant interaction of sound outcome with environmental context, $F(2,118)=46.85, p<0.001, \eta_{p}^{2}=0.44$ (also a main effect of sound outcome, $F(2,118)=8.16, p<0.001, \eta_{p}^{2}=0.12$; and no main effect of context, $F(1,59)=$ $\left.0.72, p=0.40, \eta_{p}^{2}=0.01\right) .^{2}$ This interaction shows that the way sound outcomes affected participants' judgments of whether the agent had been present differed depending on the context in which each sound outcome occurred. Next we examined our specific predictions in more detail.

${ }^{2}$ One-way repeated-measures ANOVAs within each context were included in the preregistration; so we include them here: These analyses similarly showed effects of sound outcome in the Normal Xylophone, $F(2,176)=44.43, p<0.001$, and in the Bars-scrambled-xylophone, $\mathrm{F}(2,176)=23.4, p<0.001$. 


\section{What was present when the sounds occurred?}

\section{a. Normal Xylophone}

Predictions
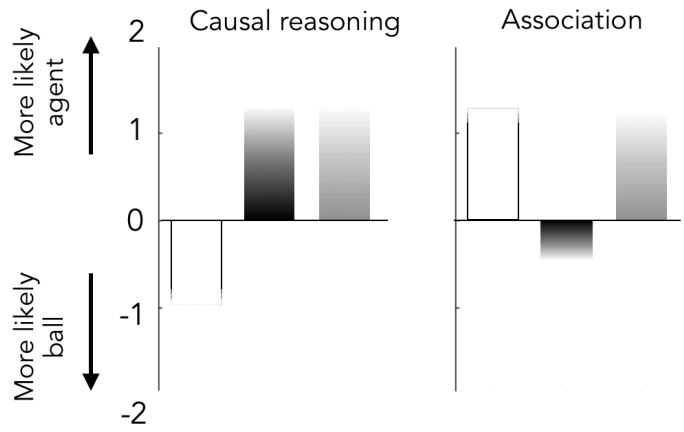

Results

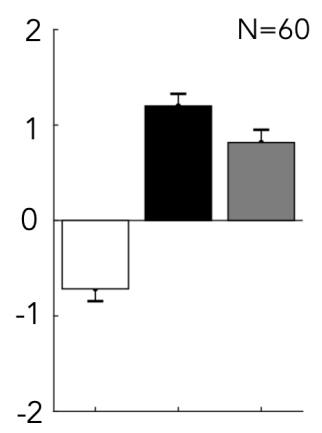

$$
\begin{array}{ll}
\text { Sound that } & \square \text { Orderly descending scale } \\
\text { occurred: } & \square \text { Disorderly scrambled tones } \\
& \square \text { Orderly ascending scale }
\end{array}
$$

\section{b. Bars-Scrambled Xylophone}

Predictions

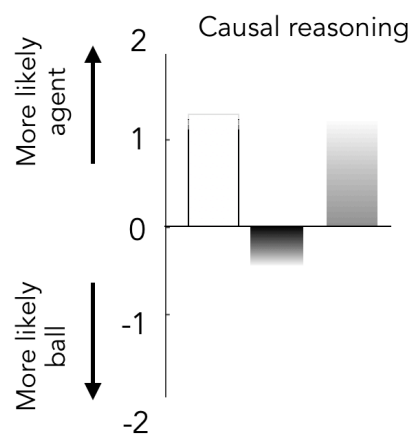

Results

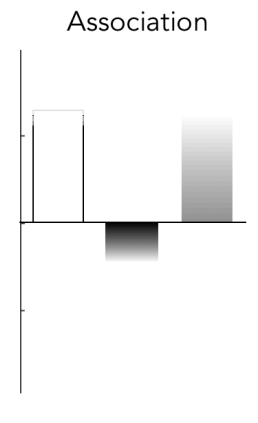

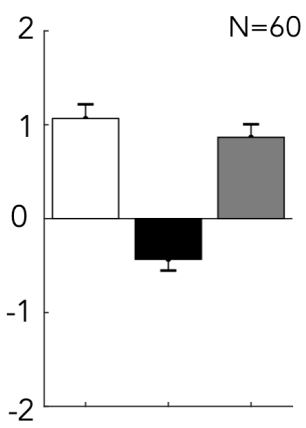

Figure 4. Predictions and results, Exp. 2. (a) In the normal xylophone context, the two accounts make distinct predictions about which sounds will lead participants to judge that an agent was present. Results are predicted only by the causal reasoning account, and show a 'flip' from what is typically reported: Disorderly scrambled tones, and not the orderly descending scale, is linked with agents. In addition, the ascending scale was not linked with agents any more than the scrambled tones - in spite of being more orderly. (b) In the scrambled xylophone context, where the descending scale could be produced by gravity, this orderly stimulus was linked with agents. The link with agents depended on the presence of an alternative physical explanation, and not on the orderliness of the outcomes.

As predicted, in the context of the normal xylophone, participants judged it more likely that the agent (versus the ball) had been present after hearing the scrambled tones 
$(M=1.2, S E M=0.14 ; t(59)=8.44, p<0.001)$, and the ascending scale $(M=0.82, t(59)$ $=4.84, p<0.001)$. After hearing the orderly descending scale, participants judged the ball more likely present $(M=-0.72, S E M=0.14 ; t(59)-5.02, p<0.001)$.

In the context of the bars-scrambled xylophone, participants' judgments flipped in the manner predicted by causal reasoning: Now that the orderly descending scale could not be produced by gravity alone, participants judged that an agent was present to produce it $(M=1.07, S E M=0.17 ; t(59)=6.23, p<0.001)$. Participants' judgments about the descending scale significantly differed across the two contexts (Mean difference=$1.78, d=-1.03 ; t(59)=-7.99, p<0.001)$. Similarly, now that the scrambled tones could be produced by gravity alone, participants did not link the scrambled tones to agents, and judged that the ball was more likely present $(M=-0.43, S E M=0.15, t(59)=-2.91, p=$ 0.005); participants' judgments about the scrambled tones significantly differed across the two contexts $($ Mean difference $=1.63, d=0.91 ; t(59)=-7.05, p<0.001)$. The ascending scale could not be produced by gravity in either context; as predicted, in the bars-scrambled context participants continued to link it with agents $(M=0.87, t(59)=$ 4.77, $p<0.001)$. Across both contexts, participants thus judged that the agent had been present when the sounds had no alternative physical explanation, and did not do so when an alternative physical explanation was possible. Even when comparing the very same sound outcome across contexts - and thus the very same level of orderliness - it was the presence or absence of alternative explanation that predicted agency judgments, not the orderliness of the sound itself.

Finally, we compared judgments regarding the two outcomes that were equated to both lack an alternative physical explanation, but differ in orderliness: The ascending 
scale and scrambled tones, in the bars-descending xylophone context. If causal reasoning and associations between the percept of order and agents both affect judgments simultaneously, then the ascending scale should cue agency more strongly than the scrambled tones (as the ascending scale is both orderly and also lacks an alternative physical explanation). In contrast to these predictions, the two conditions were not significantly different, and numerically participants were less likely to select the agent in the orderly ascending scale condition than in the scrambled tones condition (Ascending: $M=0.82, S E M=0.17$; Scrambled tones: $M=1.2, S E M=0.14, t(59)=-1.69, p=0.10)$. This suggests that associations between the percept of order and agents did not affect participants' judgments.

\section{Discussion}

This experiment addressed two outstanding questions. Firstly: Were the 'flipped' results of Experiment 1 due to the presence of an alternative physical explanation for order - or some other unknown aspect of our stimuli? We compared the normal xylophone to a new context, in which the xylophone's bars appeared in scrambled positions, as if they had been switched around. This provided an analogue to the situations explored in previous work, in which inanimate forces could not produce order (e.g. a mixed pile of blocks on a table, Newman et al., 2010): Similarly, on this new xylophone, rolling downhill would generate a scrambled sequence of tones, not an orderly descending scale. We were able to conceptually replicate previous work using our experimental paradigm: In a context where gravity could not produce orderly outcomes (bars-scrambled xylophone), the orderly stimuli led people to infer that an agent was present, while the disorderly scrambled tones did not. 
We compared this conceptual replication condition with our closely matched test condition - changing only the placement of the xylophone's bars, such that the ball could now produce the descending scale by rolling downhill, providing an alternative explanation for this orderly outcome. The typically reported link between order and agents disappeared (and again, flipped) when the context is subtly changed in this way: In the normal xylophone conditions, the very same orderly descending scale did not lead people to expect an agent, while the disorderly scrambled tones did lead people to expect an agent. The close matching of the two xylophone conditions - and contrasting results provides strong evidence that the 'flipped' results are due to the presence of an alternative physical explanation for order rather than some other unknown aspect of our stimuli.

This experiment also addressed a second outstanding question. Is it the case that both types of processes (causal reasoning, and associations between the percept of order and agents) are occurring and affecting expectations of agents simultaneously? Our findings suggest that this is not the case. Both the ascending scale and the scrambled tones lacked an alternative physical explanation in the context of the normal xylophone, because gravity would not allow the ball to produce either one. However, these outcomes differed in perceived orderliness. We found that agency judgments were equivalent for these two outcomes in the normal xylophone context: Participants did not expect an agent more strongly for the orderly outcome than the scrambled outcome. Thus, when we controlled for factors that would affect causal reasoning, perceived orderliness had no effect. These data suggest that causal reasoning about how order could be generated, not the detection of orderliness itself, drove participants to infer the presence of an agent. 


\section{General Discussion}

The first important finding from these experiments is that, contrary to claims that the link between observing order and expecting animate agents is automatic and obligatory, we found that it is instead quite malleable: It can be eliminated by fully understanding an alternative mechanism by which order could arise. Participants saw a scene involving a staircase-like xylophone, where a ball simply rolling down the xylophone would produce an orderly sound sequence - a descending scale. This provided an alternative physical-mechanical explanation for how the orderly descending scale could be generated. We found that the descending scale did not lead people to expect an agent in this context, in spite of its orderliness. In contrast, a scrambled sequence of the same tones - which would require self-propelled movement to produce in this context did lead people to expect an agent.

Across two experiments, when the environment provided a physical-mechanical explanation for an orderly sequence of sounds, people did not infer the presence of an agent after hearing the orderly outcome. When the environment was subtly changed, so that it could not provide an alternative explanation for the orderly outcome, participants readily inferred the presence of an agent from the same orderly stimulus. Overall, we find that the link between order and agency can be established or eliminated - based on the absence or presence of an alternative explanation of how order could arise.

These findings support our hypothesis about the nature of the cognitive processes that link order with agents. Only a rich, structured system of causal reasoning would allow one plausible alternative explanation to weaken evidence for other explanations (Gopnik \& Sobel, 2000; Pearl, 2000; Tenenbaum et al., 2006). People appear to link 
order with agents by reasoning about its most likely cause, using their intuitive knowledge of physical and social causality (Battaglia et al., 2013; Gelman \& Opfer, 2007; Gopnik, 2012; Spelke \& Kinzler, 2007; Tenenbaum et al., 2006).

In turn, these findings rule out an influential alternative account, providing evidence that the link between order and agents is not driven by associations with percepts of order. This account is currently seen as consistent with a broad set of existing findings on order and agency (Hamlin \& Baron, 2014; Newman \& Keil, 2015; Meng, Griffiths \& Xu, 2017), and predicts that detection of orderly stimuli should lead people to infer the presence of agents, independent of other factors. Across both experiments, while the presence or absence of an alternative explanation dramatically affected participants' agency judgments, the perceived orderliness of the outcome did not. For example, when we controlled for factors that would affect causal reasoning, and manipulated orderliness (ascending versus scrambled tones, in the normal xylophone context; Exp. 2), orderliness had no effect: Participants inferred the presence of an agent no more often for order than disorder. Our findings thus show that adult reasoning is not captured by a simple associative account.

\section{A 'default' to link order with agents}

Is there nevertheless a 'default' link between order and agency, when we lack other information? This may be the case - because order cannot arise without an agent in most situations, we may learn that agents are the most likely explanation for order, and thus use them as a 'default' in the absence of other information. This idea is consistent with a causal reasoning account: In Bayesian terms, this idea can be formalized in terms 
of priors, such that people have high priors on agents as the likely cause of order, perhaps based on previous experience with more transparent causal systems. So, our data do not speak against the idea that agents are a default explanation for order. What our data show is that the cognition underlying this link is more than a simple heuristic or automatic association - inferring agents from order is not obligatory, but can be easily overridden with other explanations when they are available. This new understanding of the malleability and cognitive basis of the link between order and agents has implications across several areas of cognitive science (see below).

There is a second plausible sense in which the order-agent link could be a 'default': As a heuristic that is used only when under cognitive load. Human cognition has been characterized as consisting of two parallel kinds of reasoning processes: One system that is slow, reasoned, rational and conscious; and one system that is fast, efficient, and uses simple heuristics (Evans, 2008; Kahneman, 2011; Stanovich, 2011). When people are put under cognitive load - either by performing additional simultaneous tasks, or by adding pressure to complete the task quickly - people often shift from rational, conscious reasoning to simpler, more biased heuristics (Kelemen, Rottman \& Seston, 2013; Strickland, Fisher, Keil \& Knobe, 2014). The current experiments suggest that under typical conditions, the link between order and agents is a product of rational inference, not simple heuristics. An important next step will be to determine whether this reasoning persists under conditions of cognitive load, or whether a simple heuristic (that order is always caused by agents) emerges when under cognitive load.

This work also raises a developmental question: Work with infants shows that the link between order and agents exists as early as the first year of life (Ma et al, 2013; Ma 
\& Xu, 2013; Newman et al., 2010; Keil \& Newman, 2015). Do infants use causal reasoning to link order with agents, similarly to adults? Or do simpler associations drive the link between order and agency in early life? Either of these accounts are possible: Observations of agents producing order begin early in life, and thus associations between order and agents could be learned in infancy (Keil \& Newman, 2015). However, infants are also capable of causal inference: Infants can understand and make predictions about physical-mechanical interactions and the behavior of animate agents (Gelman \& Opfer, 2002; Gergely et al., 1995; Kinzler \& Spelke, 2007; Teglas et al., 2011; Liu, Ullman, Tenenbaum \& Spelke, 2018), and infer the presence of agents to explain events that seem to require self-propelled movement (Saxe et al., 2005; Saxe, Tzelnic \& Carey, 2007). These systems may support adult-like causal reasoning about orderly stimuli even in infancy. Future work with infants may address whether the link between order and agents shows developmental continuity, such that causal reasoning plays a role even in early life; or shows a shift from using perceptual features and heuristics in infancy to deeper causal mechanisms later in childhood.

\section{Is evolution different? Effects of core knowledge and intuitiveness}

In these experiments, an alternative physical explanation for order eliminated the link between order and agents. Will any alternative explanation weaken the link between order and agents, or are some alternative explanations more potent than others?

Evolution by natural selection provides an informative case for contrast.

Evolution is the most salient domain in which these questions frequently arise in science and society, and have important policy implications (Bloom \& Skolnick Weisberg, 2007). Just like a xylophone and gravity provide a non-agentic explanation of orderly sounds, 
natural selection provides a non-agentic explanation of order in the biological world (Futuyma, 1998). Agentic explanations for order in nature, such as supernatural creator gods, develop early in life (Kelemen, 2004; Evans, 2000, 2001) and are notoriously sticky: Even people who have learned about natural selection often maintain creationist beliefs explicitly or implicitly (Blackwell, Powell \& Dukes, 2003; Jarnefelt, FordCanfield \& Kelemen, 2015; Kelemen, Rottman \& Seston, 2013; Shtulman \& Varcarel, 2012). What makes the physical-mechanical explanation in the current work a more potent alternative explanation for order than the process of natural selection?

One crucial difference may be whether understanding the alternative explanation relies on 'core' aspects of cognition - systems of reasoning that are early-developing in infancy, universal across culture, and shared with other species (Spelke \& Kinzler, 2007). Being grounded in core aspects of cognition may make these explanations more intuitive, allowing them to more effectively compete with agent-based explanations.

In our experiments, the alternative explanation involved physical causes that are easily parsed by our core system for physical reasoning: One object physically striking another (e.g. ball, stair), causing an effect (a sound). Understanding of physicalmechanical interactions of this kind is core to cognition in that it is universal, early developing, and shared with other species (Gelman \& Opfer, 2007; Leslie \& Keeble, 1987; Mascalzoni, Regolin \& Vallortigara, 2010; Spelke \& Kinzler, 2007; Wellman \& Gelman, 1992). In contrast, the process of natural selection is not easily parsed by this core system: The process consists of change in the population, rather than any single individual; and occurs over many generations, rather than over a single lifespan (Futuyma, 1998). This lack of fit with intuitive mental theories is thought to make 
evolution difficult to learn (Evans, 2000; Kelemen, 2012; Shtulman, 2006; 2017). We hypothesize that this lack of fit also explains why knowledge of evolution fails to completely break the link between agents and order in nature.

This idea seems plausible because these alternative explanations must 'compete' with agent-based explanations, many of which are highly intuitive and part of core cognition (Gelman \& Opfer, 2007; Spelke \& Kinzler, 2007). In particular, understanding other agents as animate and goal-directed is universal, early-developing, and shared with other species (Gergely et al., 1995; Gelman \& Opfer, 2007; Kamewari et al., 2005; Kim \& Song, 2015; Sodian, Schoppner \& Metz, 2004; Spelke \& Kinzler, 2007; Woodward, 1998). This core system is thought to play a foundational role in religious cognition by driving the formation and persistence of supernatural agent concepts and creationist beliefs about order in nature (Barrett, 2007; Bering, 2011; Cornelius \& Lopez-Mobilia, 2013; Kelemen, 1999, 2004). By hypothesis, perhaps alternative explanations can only weaken the link between order and agents when they are consistent with core knowledge. This may put this explanation on equal footing with agent-based explanations, allowing the physical explanation to compete with agents as explanations.

\section{Causal inference about sound: Implications for music cognition}

The current work also has important implications for representations of music. In music cognition, one major question of interest is why music is linked with movement: Music drives movement (Repp \& Su, 2013; Thaut et al., 1996; Schachner et al. 2009), activates motor representations in the brain (Grahn \& Brett, 2007), and is linked with movement from early in life (Hannon, Schachner \& Nave-Blodgett, 2017; Zentner \& 
ORDER, AGENTS AND ALTERNATIVE EXPLANATIONS

Eerola, 2010) and across all cultures (Brown, 1991; Sievers, Polansky, Casey, \& Wheatley 2013). Why are music and movement so closely linked?

Previous work has posited that low-level, modality-specific connections between auditory and motor representations cause this connection (Chen, Penhune \& Zatorre, 2008; Grahn \& Brett, 2007; Patel, Iversen, Chen, \& Repp, 2005). Our data suggest a novel explanation: In addition to low-level perceptual-motor links, causal reasoning may play a role. Our data show that people reasoned about the causes of musical sounds, and that their judgments were not driven by perceptual features of the sounds, but by inferences about what events in the world would cause those sounds. In this way, higherlevel cognition, not just perceptual systems, may link music with movement. By supporting abstract inferences about the actions required to cause sounds, causal reasoning may allow music to activate representations of movements in a top-down way, through high-level cognition.

The abstract, causal nature of these inferences helps inform another major question in music cognition: Why does music activate social concepts, and social cognitive brain mechanisms (Changizi, 2011; Grahn \& Brett, 2007; Launay, 2015; Overy \& Molnar-Szakaks, 2009; Sievers et al., 2012)? If people engage in causal reasoning about musical sounds, this connection is no surprise: Causal reasoning would allow people to jointly infer not only the movements that caused the sounds, but also what likely caused those movements - and thus whether an animate agent was present. In particular, if we engage in causal reasoning, sounds that would require self-propelled movement, perception, or goal-directed action to produce should lead us to infer that an 
animate agent was the cause (Gelman \& Opfer, 2007; Johnson, 2003; Gergely et al., 1995; Spelke \& Kinzler, 2007).

Our experiments provide evidence that people are making this inferential leap from sounds to agents: Based on the musical sounds they heard, people made systematic inferences about whether an animate agent was present or not. This shows that through high-level causal reasoning, music can activate abstract social concepts like agency and animacy, and lead to the belief that an agent was present to cause the sounds. This helps to explain why musical sounds may be intrinsically linked to the social domain.

Lastly, this work contributes to our understanding of auditory event perception (Bregman, 1990; Gaver, 1993), by suggesting a role of high-level causal reasoning in this process. Our experiments demonstrate that people can use pitch information, not just timing, to inform inferences about the most likely cause of sounds; and also demonstrate that causal reasoning allows for abstract inferences about whether the causes of sounds are animate or not. This lays a foundation for future work to formalize how people perceive the sources of sounds using Bayesian inference - an enterprise that is just now beginning in earnest (Seigel, Tenenbaum, \& McDermott, 2018; Gerstenberg, Siegel \& Tenenbaum, 2018).

\section{Conclusion}

Overall, we find that the link between order and agents is malleable, and can be explained away: It can be eliminated by fully understanding an alternative mechanism by which order could arise. When context provided an alternative physical explanation for an orderly outcome, people did not infer the presence of an agent from order. When the 
same orderly outcome occurred in a similar context that did not provide an alternative explanation, participants inferred the presence of an agent from the same orderly stimulus. When outcomes were equated to lack an alternative explanation, participants inferred the presence of an agent no more for order than disorder. These findings show that causal reasoning mediates the link between order and agents, and suggest that a network of learned associations between perceptual features of order and agents does not play a major role. These findings lay a foundation for better understanding of our cognitive representations of a wide range of orderly stimuli, and inform our understanding of cognition about orderly sounds like music, and order in nature.

\section{Author contributions}

AS conceived and designed the experiments. AS and MK performed the experiments, analyzed the data, and wrote the paper.

\section{Acknowledgements}

The authors thank Roman Feiman, Joshua Rottman, Yuan Meng, Susan Carey, Deborah Kelemen, Timothy Brady, Jamie Fehribach, Mio Shang, and Arthur Fu for valuable feedback and comments on the manuscript.

\section{Declaration of Conflicting Interests}

The author declares that there were no conflicts of interest with respect to authorship or the publication of this article. 
ORDER, AGENTS AND ALTERNATIVE EXPLANATIONS

\section{Funding}

This work was supported in part by Grant No. F32HD075570 from the Eunice

Kennedy Shriver National Institute of Child Health and Human Development, and by Grant No. BCS-1749551 from the National Science Foundation to Adena Schachner. 


\section{References}

Baer, C., Tam, C., Nancekivell, S., \& Friedman, O. (2013). Order and disorder in children's ownership judgments. Poster presented at the meeting of the Society for Research in Child Development, Seattle, WA.

Baker, C. L., Saxe, R. R., \& Tenenbaum, J. B. (2009). Action understanding as inverse planning. Cognition, 113(3), 329-349. doi:10.1016/j.cognition.2009.07.005

Barrett, J. L. (2007). Cognitive science of religion: What is it and why is it? Religion Compass, 1, 768-786. doi:10.1111/j.1749-8171.2007.00042.x

Battaglia, P. W., Hamrick, J. B., \& Tenenbaum, J. B. (2013). Simulation as an engine of physical scene understanding. Proceedings of the National Academy of Sciences, 110(45), 18327-18332. http://doi.org/10.1073/pnas.1306572110

Bering, J. (2011). The Belief Instinct: The Psychology of Souls, Destiny, and the Meaning of Life. New York: W.W. Norton \& Co.

Berinsky, A. J., Huber, G. A., \& Lenz, G. S. (2012). Evaluating Online Labor Markets for Experimental Research: Amazon.com's Mechanical Turk. Political Analysis, 20(3), 351-368. doi:10.1093/polana/mpr057/-/DC1

Blackwell, W.H., Powell, M.J., \& Dukes, G.H. (2003). The problem of student acceptance of evolution. Journal of Biological Education, 37(2), 58-67. DOI: $10.1080 / 00219266.2003 .9655852$

Bloom, P., \& Skolnick Weisberg, D. (2007). Childhood Origins of Adult Resistance to Science. Science, 316 (5827), 996-997.

DOI: 10.1126/science. 1133398 
ORDER, AGENTS AND ALTERNATIVE EXPLANATIONS

Bregman, A.S. (1990). Auditory scene analysis: The perceptual organization of sound. Cambridge, MA: MIT Press.

Brown, D. E. (1991). Human Universals. New York: McGraw-Hill.

Changizi, M. (2011). Harnessed: How Language and Music Mimicked Nature and Transformed Ape to Man. Dallas, TX: BenBalla Books.

Chen, J. L., Penhune, V. B., \& Zatorre, R. J. (2008). Listening to musical rhythms recruits motor regions of the brain. Cerebral Cortex, 18(12), 2844-2854.

Cornelius, C., \& Lopez-Mobilia, G. (2013). Relations between anthropomorphism, predictability, and patterned outcomes. Poster presented at the Eighth Biennial meeting of the Cognitive Development Society, Memphis, TN.

Cross, I. (2003). Music as a biocultural phenomenon. Annals of the New York Academy of Sciences, 999, 106-111.

Dawkins, R. (1986). The Blind Watchmaker. New York: W.W. Norton \& Co.

Dawkins, R. (2006). The God Delusion. Boston: Houghton Mifflin.

Evans, E.M. (2000). Beyond Scopes: Why Creationism is here to stay. In K.S. Rosengren, C.N. Johnson, \& P.L. Harris (Eds.), Imagining the impossible: The development of magical, scientific and religious thinking in contemporary society (pp. 305-333). Cambridge, England: Cambridge University Press.

Evans, E.M. (2001). Cognitive and contextual factors in the emergence of diverse belief systems: Creation versus evolution. Cognitive Psychology, 42, 217-266.

Evans, J. S. B. (2008). Dual-processing accounts of reasoning, judgment, and social cognition. Annu. Rev. Psychol., 59, 255-278. 
ORDER, AGENTS AND ALTERNATIVE EXPLANATIONS

Friedman, W. J. (2001). The development of an intuitive understanding of entropy. Child Development, 72, 460-473.

Futuyma, D. J. (1998). Evolutionary biology (3rd ed.). Sunderland, MA: Sinauer Associates.

Gaver, W.W. (1993b). What in the world do we hear? An ecological approach to auditory event perception. Ecological Psychology, 5, 1-29.

Gerstenberg, T., Siegel, M., \& Tenenbaum, J. (2018). What happened? Reconstructing the past through vision and sound. Talk presented at the Fortieth meeting of the Cognitive Sciences Society, Madison, W.I.

Gelman, S. A., \& Opfer, J. E. (2007). Development of the animate-inanimate distinction. Blackwell Handbook of Childhood Cognitive Development, 151-166.

Gergely, G., Nádasdy, Z., Csibra, G., \& Bíró, S. (1995). Taking the intentional stance at 12 months of age. Cognition, 56, 165-193.

Goodman, J. K., Cryder, C. E., \& Cheema, A. (2012). Data Collection in a Flat World: The Strengths and Weaknesses of Mechanical Turk Samples. Journal of Behavioral Decision Making, 26(3), 213-224. doi:10.1002/bdm.1753

Gopnik, A. (2012). Scientific thinking in young children: theoretical advances, empirical research, and policy implications. Science (New York, N.Y.), 337(6102), 1623-7. http://doi.org/10.1126/science. 1223416

Gopnik, A., \& Sobel, D.M. (2000). Detecting Blickets: How young children use information about novel causal powers in categorization and induction. Child Development, 71(5), 1205-1222. 
ORDER, AGENTS AND ALTERNATIVE EXPLANATIONS

Gould, S. J. (2002). Rocks of Ages: Science and Religion in the Fullness of Life. Random House.

Grahn, J. A., \& Brett, M. (2007). Rhythm and beat perception in motor areas of the brain. Journal of cognitive neuroscience, 19(5), 893-906.

Griffiths, T. L., Daniels, D., Austerweil, J. L., \& Tenenbaum, J. B. (2018). Subjective randomness as statistical inference. Cognitive psychology, 103, 85-109.

Hamlin, J. K., \& Baron, A. S. (2014). Agency attribution in infancy: Evidence for a negativity bias. PLoS ONE, 9(5). http://doi.org/10.1371/journal.pone.0096112

Hannon, E. E., Schachner, A., \& Nave-Blodgett, J. E. (2017). Babies know bad dancing when they see it: older but not younger infants discriminate between synchronous and asynchronous audiovisual musical displays. Journal of Experimental Child Psychology, 159, 159-174.

Johnson, S. C. (2003). Detecting agents. Philosophical Transactions of the Royal Society B: Biological Sciences, 358(1431), 549-559. doi:10.1098/rstb.2002.1237

Jarnefelt, E., Ford Canfield, C. \& Kelemen, D. (2015). The divided mind of a disbeliever: Intuitive beliefs about nature as purposefully created among different groups of non-religious adults. Cognition, 140, 72-88.

Kahneman, D. (2011) Thinking, fast and slow. New York, NY: Farrar, Straus and Giroux. Keil, F. C., \& Newman, G. E. (2015). Order, order everywhere, and only an agent to think: The cognitive compulsion to infer intentional agents. Mind and Language, 30(2), 117-139. http://doi.org/10.1111/mila.12074 
ORDER, AGENTS AND ALTERNATIVE EXPLANATIONS

Kelemen, D. (2004). Are children “intuitive theists?” Reasoning about purpose and design in nature. Psychological Science, 15(5), 295-301. doi:10.1111/j.09567976.2004.00672.x

Kelemen, D. (2012). Teleological minds: How natural intuitions about agency and purpose influence learning about evolution. In K. S. Rosengren, S. K. Brem, E. M. Evans \& G. M. Sinatra (Eds.), Evolution challenges: Integrating research and practice in teaching and learning about evolution. Oxford: Oxford University Press.

Kelemen, D., Rottman, J. \& Seston, R. (2013). Professional physical scientists display tenacious teleological tendencies. Purpose-based reasoning as a cognitive default. Journal of Experimental Psychology: General, 142(4), 1074-1083.

Kim, E. Y., \& Song, H. (2015). Six-month-olds actively predict others' goal-directed actions. Cognitive Development, 33, 1-13.

Kuwabara, M., \& Smith, Li. B. (2012). Cross-cultural differences in cognitive development: Attention to relations and objects. Journal of Experimental Child Psychology, 113(1), 20-35.

Launay, J. (2015). Musical sounds, motor resonance and agency detection. Empirical Musicology Review, 10(1), 30-40.

Leslie, A. M., \& Keeble, S. (1987). Do six-month-old infants perceive causality? Cognition, 25(3), 265-288.

Lipton, P. (2004). Inference to the Best Explanation, 2nd edition. Routledge Publishers. 
ORDER, AGENTS AND ALTERNATIVE EXPLANATIONS

Liu, S., Ullman, T. D., Tenenbaum, J. B., \& Spelke, E. S. (2017). Ten-month-old infants infer the value of goals from the costs of actions. Science, 358(6366), 1038-1041. http://doi.org/10.1126/science.aag2132

Ma, L., \& Xu, F. (2013). Preverbal infants infer intentional agents from the perception of regularity. Developmental Psychology, 49(7), 1330-1337. doi:10.1037/a0029620

Ma, L., Berthiaume, V., Hoch, J., \& Xu, F. (2013). Twelve-Month-Old Infants Infer Intentional Agents from the Perception of Auditory Regularity. Poster presented at the 8th Biennial Meeting of the Cognitive Development Society, Memphis, TN.

Mascalzoni, E., Regolin, L., \& Vallortigara, G. (2010). Innate sensitivity for selfpropelled causal agency in newly hatched chicks. Proceedings of the National Academy of Sciences, 107(9), 4483-4485. http://doi.org/10.1073/pnas.0908792107

Mason, W., \& Suri, S. (2011). Conducting behavioral research on Amazon's Mechanical Turk. Behavior Research Methods, 44(1), 1-23. doi:10.3758/s13428-011-0124-6

Meng, Y., Griffiths, T. L., \& Xu, F. (2017). Inferring Intentional Agents From Violation of Randomness. Proceedings of the Cognitive Sciences Society, 2699-2704.

Newman, G. E., Keil, F. C., Kuhlmeier, V. A., \& Wynn, K. (2010). Early understandings of the link between agents and order. Proceedings of the National Academy of Sciences, 107(40), 17140-17145.

Overy, K., \& Molnar-Szakacs, I. (2009). Being Together in Time: Musical Experience and the Mirror Neuron System. Music Perception, 26(5), 489-504.

Paley, W. (1802). Natural Theology: Or, Evidences of the Existence and Attributes of the Deity, Collected from the Appearances of Nature. Philadelphia: H. Maxwell. 
ORDER, AGENTS AND ALTERNATIVE EXPLANATIONS

Patel A.D., Iversen J.R., Chen Y., \& Repp B.H. (2005) The influence of metricality and modality on synchronization with a beat. Experimental Brain Research, 163(2), $226-238$.

Pearl J. (2000.) Causality: Models, Reasoning, and Inference. Cambridge, UK: Cambridge Univ. Press

Reimers, S., Donkin, C., \& Le Pelley, M. E. (2018). Perceptions of randomness in binary sequences: Normative, heuristic, or both?. Cognition, 172, 11-25.

Repp, B. H., \& Su, Y. H. (2013). Sensorimotor synchronization: a review of recent research (2006-2012). Psychonomic Bulletin \& Review, 20(3), 403-452.

Saxe, R., Tenenbaum, J.B., \& Carey, S. (2005). Secret agents: Inferences about hidden causes by 10-and 12-month-old infants. Psychological Science, 16(12), 995.

Saxe, R. R., Tzelnic, T., \& Carey, S. (2007). Knowing who dunnit: Infants identify the causal agent in an unseen causal interaction. Developmental Psychology, 43(1), $149-158$.

Siegel, M., Tenenbaum, J \& McDermott, J. (2018). Physical Inference for Object Perception in Complex Auditory Scenes. Talk presented at the Fortieth meeting of the Cognitive Sciences Society, Madison, W.I.

Sievers, B., Polansky, L., Casey, M., \& Wheatley, T. (2012). Music and movement share a dynamic structure that supports universal expressions of emotion. Proceedings of the National Academy of Sciences, 110(1), 70-75.

Shtulman, A. (2006). Qualitative differences between naïve and scientific theories of evolution. Cognitive Psychology, 52, 170-194. 
Shtulman, A. (2017). Scienceblind: Why Our Intuitive Theories About the World Are So Often Wrong. NY: Basic Books.

Shtulman, A., \& Valcarcel, J. (2012). Scientific knowledge suppresses but does not supplant earlier intuitions. Cognition, 124(2), 209-215. http://doi.org/10.1016/j.cognition.2012.04.005

Sodian, B., Schoeppner, B., \& Metz, U. (2004). Do infants apply the principle of rational action to human agents? Infant Behavior and Development, 27(1), 31-41.

Spelke, E. S., \& Kinzler, K. D. (2007). Core knowledge. Developmental Science, 10(1), 89-96.

Stanovich, K.E. (2011). Rationality and the reflective mind. New York, NY: Oxford University Press.

Strickland, B., Fisher, Knobe, J. \& Keil, F. (2014). Syntax and intentionality: An automatic link between language and theory-of-mind. Cognition, 133(1), 249261. doi:10.1016/j.cognition.2014.05.021.

Swinburne, R. G. (1968). The argument from design. Philosophy, 43(165), 199-212.

Teglas, E., Vul, E., Girotto, V., Gonzalez, M., Tenenbaum, J. B., \& Bonatti, L. L. (2011). Pure Reasoning in 12- Month-Old Infants as Probabilistic Inference. Science, $332(6033), 1054-1059$.

Tenenbaum, J. B., Griffiths, T. L., \& Kemp, C. (2006). Theory-based Bayesian models of inductive learning and reasoning. Trends in Cognitive Sciences, 10, 309-318.

Thaut, M. H., McIntosh, G. C., Rice, R. R., Miller, R. A., Rathbun, J., \& Brault, J. M. (1996). Rhythmic auditory stimulation in gait training for Parkinson's disease patients. Movement disorders, 11(2), 193-200. 


\section{ORDER, AGENTS AND ALTERNATIVE EXPLANATIONS}

Vaesen, K. (2012). The cognitive bases of human tool use. Behavioral and Brain Sciences, 1-16. doi:10.1017/S0140525X11001452

Wellman, H.M. \& Gelman, S.A. (1992). Cognitive development: Foundational theories of core domains. Annual Review of Psychology, 43, 337-75.

Woodward, A. L. (1998). Infants selectively encode the goal object of an actor's reach. Cognition, 69(1), 1-34.

Zentner, M., \& Eerola, T. (2010). Rhythmic engagement with music in infancy. Proceedings of the National Academy of Sciences, 107(13), 5768-5773. 